\title{
Distributed sampling for rational approximation of the acoustic scattering of an airfoil
}

\author{
Francesca Bonizzoni $^{1 *}$ and Davide Pradovera ${ }^{2 * *}$ \\ ${ }^{1}$ Faculty of Mathematics, Universität Wien, Oskar-Morgenstern-Platz 1, 1090 Wien, Austria \\ ${ }^{2}$ CSQI - MATH, École Polytechnique Fédérale de Lausanne, Station 8, CH-1015 Lausanne, Switzerland
}

\begin{abstract}
In this paper we compute a reduced order model for a time-harmonic external acoustic scattering problem with parametric frequency. The employed technique is minimal rational interpolation, an explicit moment-matching method for Hilbert spacevalued meromorphic maps. We study the approximation and stability properties of this technique for different choices of the sample point set, namely fully distributed in the parameter range, and partially and fully confluent. The proposed technique is also compared with an implicit multi moment-matching method based on Galerkin projection.
\end{abstract}

\section{Problem framework}

Let $D \subset \mathbb{R}^{2}$ be the section of a plane wing with open flap (rigid and single slotted). We assume a plane acoustic wave $u_{\text {inc }}$ with unit amplitude and frequency $f_{\text {inc }}=2.5 \mathrm{kHz}$ to impinge on the wing and scatter off of it in the surrounding air $\left(c_{a i r}=343 \mathrm{~m} / \mathrm{s}\right)$. In particular, the source of the wave is located above and in front of the leading edge of the airfoil, so that the wavevector has an inclination of $\theta=45^{\circ}$ with respect to the chord line of the wing. Due to the presence of the flap slot in the main wing body, we may expect a resonance-like behavior of the scattered wave in that region. To investigate the impact of such (potential) resonance, we consider the frequency response of the pressure field around the airfoil.

Given an open finite computational domain $\Omega \subset \mathbb{R}^{2}$, with $\bar{D} \subset \Omega$, (in our experiments we choose a disk with sufficiently large radius) the scattered pressure field can be computed by solving a time-harmonic wave equation [4]:

$$
\begin{cases}-c_{\text {air }}^{2} \Delta u(f)-4 \pi^{2} f^{2} u(f)=0 & \text { in } \Omega \backslash \bar{D}=: \Omega^{\star} \\ u(f)+u_{i n c}=0 & \text { on } \partial D \\ c_{\text {air }} \partial_{n} u(f)-2 \pi \iota f u(f)=0 & \text { on } \partial \Omega\end{cases}
$$

$\iota$ being the imaginary unit. A Sommerfield radiation condition is imposed at infinity for closure of the problem. A numerical solution of problem (1) is obtained via the Finite Element (FE) method on a regular triangulation, see Figure 1. A sample of the FE pressure field is shown in Figure 2. A resonance-like behavior can be observed, since the scattered wave has peaks with pressure magnitude 5-6 times larger than the amplitude of the incoming wave.

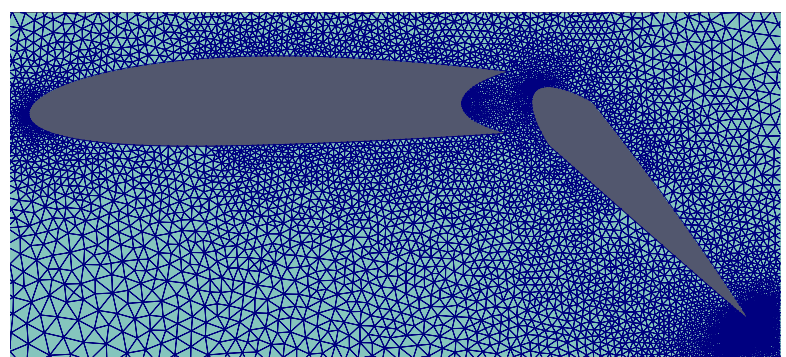

Fig. 1: Discretized computational domain near $D$. The mesh has been coarsened by a factor $\sim 3$ for visualization purposes.

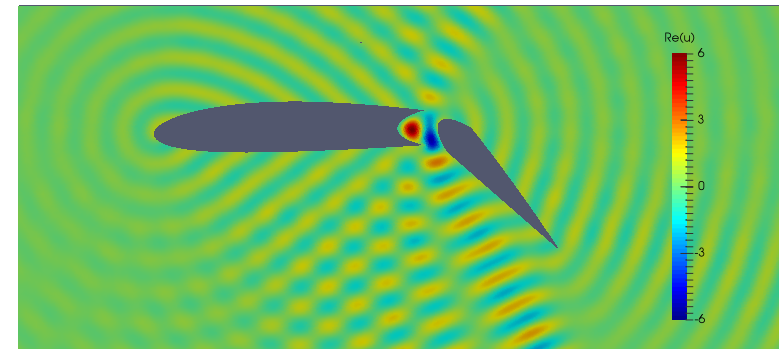

Fig. 2: Real part of the FE scattered pressure field $u(f=3 \mathrm{kHz})$. The impinging wave comes from the top-right with $45^{\circ}$ inclination.

We formalize our frequency response approximation problem as follows: given the frequency range $K=[1.8,3.2] \mathrm{kHz}$, we consider the task of approximating the solution map of (1), namely $u: \mathbb{C} \supset K \ni f \mapsto u(f) \in H^{1}\left(\Omega^{\star}\right)$ such that $u(f)$ solves (1).

\footnotetext{
* E-mail: francesca.bonizzoni@univie.ac.at. F. Bonizzoni has been supported by the FWF Firnberg-Program, grant T998, and acknowledges partial support from the Austrian Science Fund (FWF) through project F 65.

** Corresponding author. E-mail: davide.pradovera@epfl.ch. D. Pradovera has been funded by the Swiss National Science Fund through project 182236.
} 


\section{Rational model reduction}

In [1] the solution map $u$ was proven meromorphic, i.e. it can be expressed over any compact subset of $\mathbb{C}$ as the ratio between a holomorphic $H^{1}\left(\Omega^{\star}\right)$-valued function and a $\mathbb{C}$-valued polynomial of finite degree. Accordingly, we choose to approximate $u$ over $K$ by minimal rational interpolants [3]. The data needed to compute such approximant is a set of $S$ samples of the solution map, each obtained as FE solution of problem (1), at given sample points $Z_{S} \subset K$. (In particular, if a sample point $z \in Z_{S}$ appears $k>1$ times, the input samples include the FE approximations of $u(z)$ and of the $k-1$ derivatives $u^{\prime}(z), \ldots, u^{(k)}(z)$, see [1].) The approximation of the solution map $u$ yielded by this technique is a rational map of type $[M / N]$ (with $\max \{M, N\}<S$ ), i.e. the ratio of two polynomials, the numerator being $H^{1}\left(\Omega^{\star}\right)$-valued with degree $M$, and the denominator being $\mathbb{C}$-valued with degree $N$, respectively. In particular, the roots of the denominator approximate the $N$ resonances of (1) which are closest to the given parameter range.

\subsection{Numerical results}

In our numerical experiments we compute the rational approximation of $u$ via the minimal rational interpolant technique with three different choices of sample points $Z_{S}$, the cardinality of $Z_{S}$ being $S=30$ : (a) $Z_{S}^{(a)}$ are the $S$ Chebyshev nodes of $K$ (the roots of the Chebyshev polynomial of degree $S$ over $K$ ), (b) $Z_{S}^{(b)}$ are the $S / 5$ Chebyshev nodes of $K$, each considered 5 times, (c) $Z_{S}^{(c)}$ is the mid-point of $K$, considered $S$ times. In all cases we set $M=N=S-1$.

The $H^{1}\left(\Omega^{\star}\right)$-norms (weighted by $\left.f[1]\right)$ over $K$ of the surrogate solution maps are shown in Figure 3 . The approaches appear to be ordered according to their effectiveness, with (c) showing particularly unstable results. This can be explained by the ill-conditioning of the SVD problem introduced to obtain the approximant relying on fully confluent samples $Z_{S}^{(c)}$.

In Figure 4 we show the relative approximation error over $K$. The location of the samples can be clearly identified for approaches (a) and (b). We compare our technique with an implicit multi-moment-matching (MMM) method [2] based on exactly the same samples of $u$. For distributed samples, minimal rational interpolants appear to perform slightly better than MMM, while in the centered case (c) the latter approach (which simplifies to a Krylov subspace method) is the obvious winner.

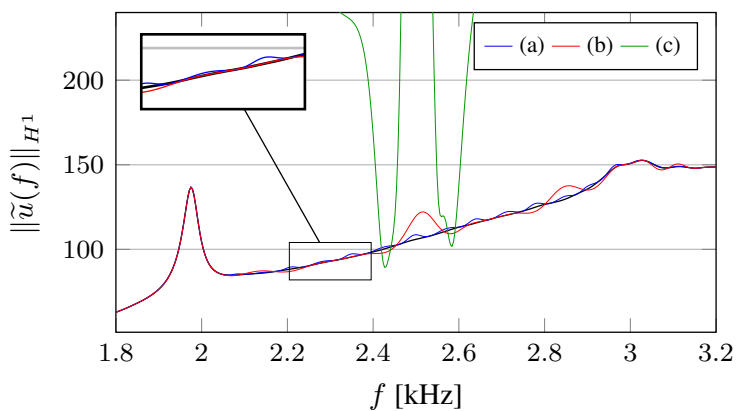

Fig. 3: Frequency-weighted $H^{1}\left(\Omega^{\star}\right)$-norm of the surrogate solution map over $K$. In black the norm of $u$.

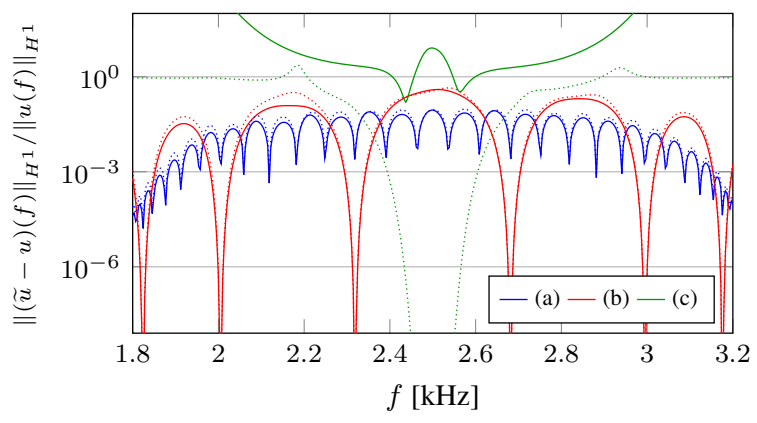

Fig. 4: Relative approximation error in the $f$-weighted $H^{1}\left(\Omega^{\star}\right)$ norm over $K$. The dotted lines show the error achieved by MMM.

\section{Conclusions}

We have shown the effectiveness of the minimal rational interpolation technique in approximating the solution map of an external scattering problem with parametric wavenumber. In particular, we have illustrated how problems may arise if too many sample points coincide (i.e. if too many derivatives are employed), since conditioning issues prevent good approximation properties. Our numerical results show that a sample set without confluence achieves the best approximation error in the $L^{\infty}(K)$-metric, whereas a partially confluent sample set yields more accurate results close to the samples, thanks to the local moment-matching property. The comparison of our method with a state-of-the-art technique has produced satisfactory results.

Acknowledgements The authors thank Fabio Nobile and Ilaria Perugia for their valuable remarks on a preliminary version of this paper.

\section{References}

[1] F. Bonizzoni, F. Nobile, I. Perugia, and D. Pradovera, ArXiv e-prints arXiv: 1805.05031 (2018).

[2] L. Feng, and P. Benner, in: Reduced Order Methods for modeling and computational reduction, edited by A. Quarteroni, and G. Rozza, MS\&A Series (Springer, Cham, 2014), vol. 9, chap. 6.

[3] D. Pradovera, ArXiv e-prints arXiv:1905.12954 (2019).

[4] M. C. Junger, and D. Feit, Sound, structures, and their interaction (MIT Press, Cambridge, 1986), chap. 10. 